\title{
QS能
}

Luis Ramón Yanguas Morte*

Ángela Andrea Caviedes Conde**

\section{IMPACTO DE LAS INVERSIONES PRIVADAS EN EL DESARROLLO DE LOS PAÍSES EN DESARROLLO}

El acceso a recursos financieros para los países en desarrollo en el siglo XXI es complicado. Los actuales desafíos de crecimiento de población, erradicación de la pobreza y cambio climático hacen que los instrumentos financieros tradicionales e innovadores sean insuficientes para cerrar la brecha de financiación del desarrollo. La inversión extranjera directa continúa siendo uno de los principales instrumentos. Este artículo describe su evolución y características, presentando un modelo econométrico descriptivo de los factores determinantes de sus flujos de entrada por región y tipo de país. Las condiciones macroeconómicas, la apertura y política comerciales muestran la mayor influencia y significación.

Palabras clave: inversión directa extranjera, financiación del desarrollo.

Clasificación JEL: F10, F63, O10.

\section{Introducción}

En este trabajo ${ }^{1}$ nos proponemos presentar algunas de las características de las inversiones directas empresariales de los países en desarrollo. Para ello lo dividiremos en varias partes: en la primera se repasan algunas de las características del desarrollo económico y de los instrumentos clásicos de financiación del mismo. En una segunda parte estudiaremos

*Director de finanzas. BCD TRAVEL, Atlanta, GA, Estados Unidos. Doctor por la Universidad de Alcalá. CPA, CMA.

**Profesora de Marketing y Negocios Internacionales. Universidad Internacional de la Rioja. Doctora por la Universidad de Alcalá.

Versión de septiembre de 2017.

1 Está basado en la tesis doctoral La financiacion internacional a los países en desarrollo en el siglo XXI, realizada por el doctor Luis Yanguas y dirigida por los doctores Miguel Ángel Díaz Mier y Ángela Andrea Caviedes Conde. las características de la inversión directa extranjera (IDE) a nivel regional. Finalmente se presenta un modelo econométrico para valorar las situaciones de tales países.

\section{Conceptos}

Para analizar las características del desarroIlo, un primer paso es entender las dualidades que encontramos respecto a su naturaleza:

En primer lugar, se trata tanto de un derecho como de una obligación. Es un derecho enfocado a las personas, en un proceso orientado a mejorar el bienestar de la población y de todos los individuos, que son participantes activos, libres y protagonistas de su propio $\triangle$ 
proceso de desarrollo y de la justa distribución de sus beneficios, tal y como se reconoce en la Declaración de Naciones Unidas del derecho al desarrollo de 1986.

En cuanto a obligación, el desarrollo comprometería a todas las partes involucradas, sean países donantes, economías en desarroIlo, empresas multinacionales, sociedades o individuos; porque lo importante es que todos tenemos una responsabilidad con los resultados del proceso mundial de desarrollo.

En segundo lugar, se pueden considerar tanto las causas como los efectos del desarrollo. Entre las causas se encuentran las que ha estudiado la sucesión de teorías económicas que han intentado explicar y predecir la realidad del desarrollo. Así, la agenda institucional del desarrollo reflejaría el compromiso de los Gobiernos y los consumidores para mejorarlo. Entre los efectos han de estudiarse los constantes cambios en la realidad política, económica, social y cultural, hasta cierto punto, impactadas por las acciones de los Gobiernos y las comunidades.

En tercer lugar, el desarrollo se puede analizar tanto desde una perspectiva global como local. Por un lado, está sometido a las fuerzas de transformación globales (tales como el crecimiento y envejecimiento de la población mundial, el crecimiento de la clase media mundial, el cambio climático, la crisis internacional y el desempleo, la globalización, la financiación y las migraciones internacionales, por nombrar las más importantes) y desde la perspectiva local, a las dinámicas de las comunidades e individuos. Ambas perspectivas están transformando el mundo tal como lo conocemos en un proceso continuo e incesante.

Otra característica importante del desarrollo es el creciente grado de complejidad del mismo. La variedad de circunstancias específicas y diferentes que enfrenta cada país en desarrollo hace que el proceso de desarrollo sea complicado y no pueda ser abordado con soluciones estándares, sino que sean necesarias soluciones múltiples y específicas a la medida de las circunstancias y los problemas de cada país, en tanto va avanzando por cada uno de los diferentes estados de desarrollo.

Finalmente, existe en la actualidad un mayor grado de conciencia en el tema del desarrollo, en parte debido al efecto de la tecnología y la mayor interconexión entre personas y comunidades, y por otra parte porque ya se empiezan a ver consecuencias negativas en el planeta debido al cambio climático.

Para completar estos elementos necesarios para nuestro análisis se necesita añadir las perspectivas del desarrollo desde la posición de un país en desarrollo, presentando una serie de factores que ayudan a explicar el estado de su situación.

El primero es la influencia de la herencia histórica como resultado de los procesos de descolonización (ya fuera este pacífico o violento) y las posteriores relaciones con la potencia colonizadora.

El segundo está relacionado con la estructura de dicho país, resultado de una combinación de características como son el tamaño (geográfico y poblacional), los recursos naturales (agua, petróleo, gas...) y su localización (sin acceso al mar, islas, playas, etcétera).

Un tercer factor se relaciona con sus capacidades, definidas por sus instituciones y sistemas políticos y la habilidad del Gobierno en la gestión de las estrategias y políticas de desarrollo.

Presentadas estas características, hemos de resaltar que el siglo xxı es testigo de un momento sin parangón del desarrollo en el que se enfrenta a los mayores desafíos de su historia, debido principalmente a tres razones. 
La primera es la combinación de las fuerzas de transformación global como son el crecimiento y envejecimiento de la población mundial (explosión e implosión), el desempleo, las migraciones, etcétera, que suponen una carga para los países en su camino al desarrollo.

En segundo lugar, varios años antes del cumplimiento, en 2015, de los objetivos de desarrollo del milenio, la agenda del desarrollo de las Naciones Unidas post 2015 comenzó como un proceso inclusivo para reforzar la alianza global del desarrollo, llevar a cabo un plan específico por los países menos desarrollados, erradicar la pobreza sin dejar a nadie atrás y mantener el desarrollo sostenible como núcleo central, con el objetivo de comprometerse a mejorar sustancialmente la reducción de pobreza, sostenibilidad ambiental (cambio climático y protección de la atmósfera, bosques y océanos), educación, acceso a la energía, agua y servicios sanitarios.

En tercer lugar, en la conferencia de cambio climático de Naciones Unidas de París en 2015, los países asistentes, entre los que se encontraban una mayoría de países en desarrollo, acordaron cumplir con el objetivo de mantener el crecimiento de la temperatura desde la era preindustrial en 1,5 grados centígrados, lo que significaría mantener cero emisiones de dióxido de carbono y otros gases alrededor de 2030.

La situación económica mundial en este siglo muestra actualmente signos de débil crecimiento del producto y las perspectivas en 2017 son aún débiles, debido al bajo nivel de precios de las materias primas (combustibles, metales, etcétera), a las condiciones financieras externas todavía restrictivas, a la caída de los flujos de capital que están teniendo un impacto sobre el crecimiento económico (por ejemplo, el caso de China o la caída del PIB en Latinoamérica, el modesto crecimiento en Oriente Medio y el crecimiento gradual de África Subsahariana, por nombrar algunas zonas).

Se espera que la recuperación económica se produzca de manera desigual y de forma modesta en las economías desarrolladas (como la UE y Japón, que continúan con sus políticas monetarias restrictivas). En este contexto, nos preguntamos si los instrumentos financieros internacionales serán capaces de proveer de los fondos necesarios para asegurar que los recursos se movilicen allá donde sea necesario para cumplir con los compromisos de desarrollo. Recordemos que los principales instrumentos que se suelen analizar son los superávits del comercio de bienes y servicios, los créditos a la exportación, la ayuda oficial (AOD), la asistencia técnica oficial y la deuda externa. A ello cabe añadir desde una perspectiva privada las remesas de emigrantes y, sobre todo, la inversión extranjera directa a la que dedicamos este trabajo.

Ahora bien, conviene destacar la existencia de una brecha entre los objetivos comprometidos y los recursos disponibles para financiarla. Ello es, por desgracia, una constante en la historia del desarrollo que ha venido minando los esfuerzos llevados a cabo en la comunidad global. A pesar del gran crecimiento mostrado durante los últimos cincuenta años en los flujos movilizados a través de los instrumentos financieros señalados y del crecimiento de los compromisos de los países donantes, la brecha de la financiación continúa siendo creciente.

Otro aspecto que empieza a tener atención es el referido a los que se denominan instrumentos innovadores de financiación que podrían jugar un papel importante para la superación de la citada brecha. Son considerados instrumentos innovadores varios tipos: mecanismos de garantías, mecanismos de mercado, swaps de deuda, impuestos sobre actividades globales, contribuciones $\triangleright$ 
de ciudadanos (individuos y empresas), loterías y otros. Más de veinte países han puesto en marcha uno o más instrumentos financieros innovadores. Hasta la fecha, y gracias a esos mecanismos, se han recaudado más de seis mil millones de dólares desde 2006, si bien la falta de un acuerdo global respecto a su ámbito de aplicación y cuantía viene limitando su impacto total.

Por ello, los instrumentos de financiación tradicionales continúan siendo la principal fuente financiera del desarrollo disponible para los países menos adelantados en el contexto actual en que el desarrollo se enfrenta a los mayores desafíos de la historia. Como señalamos, la recuperación económica todavía es débil y desigual y los instrumentos de financiación innovadora todavía no tienen suficiente apoyo y volumen para tener un impacto considerable.

En este artículo vamos a analizar en detalle la IED, categoría de inversiones que se define como la acción de un residente sobre el control o el alto grado de influencia en la gestión de una empresa de otra economía, y en nuestro caso un país en desarrollo. Igualmente se ha definido la IED, de acuerdo con el informe Balance of Payments and International Investment (FMI, 2009), como la situación en que la propiedad otorga control o influencia. La inversión directa tiene algunas excepciones (inversiones en empresas controladas o influenciadas de manera indirecta, inversiones en empresas amigas, inversiones de deuda o reserva).

Desde nuestro punto de vista las principales razones para el estudio de la IDE son las siguientes: 1) que proporciona al país receptor fondos para inversiones productivas; 2) que es más estable que otros instrumentos por dirigirse normalmente a un mayor largo plazo;
3) porque la introducción de nuevos productos o procesos productivos tiene efectos multiplicadores en la tecnología y el conocimiento; 4) por incentivar la competitividad de las empresas locales; 5) por incrementar la productividad en el mercado local; y 6) por incrementar los salarios y rentas de la población local.

\section{Principales características de la IED en las últimas décadas}

En los últimos decenios la IED ha crecido significativamente debido principalmente a una serie de factores, como son: 1) la reducción de los costes de transportes y comunicaciones, la liberalización de los mercados financieros y los acuerdos de integración de comercio a nivel regional; 2) la liberalización económica, las privatizaciones de empresas estatales y los cambios en las regulaciones de los acuerdos de inversiones internacionales; 3) el desarrollo del sector de servicios y la liberalización de sectores como telecomunicaciones, banca, finanzas, asociadas en su estudio a las cadenas de valor; 4) el rápido crecimiento de los flujos de IED hacia los países en desarrollo y los menos adelantados; y, finalmente, 5) la explosión de las operaciones de fusiones y adquisiciones de deuda a través de los mercados internacionales de capital.

Adicionalmente, se observa cómo la IED está concentrada de manera significativa. Los flujos netos de salidas se centran en torno a un número reducido de países emisores fundamentalmente desarrollados y principalmente de miembros de la OCDE, lo cual incrementa su sensibilidad respecto a la evolución de un número reducido de economías y explica sus tasas recesivas en períodos de crisis mundiales. Por otro lado, a nivel de flujos $\triangleright$ 
de entrada también se da esa concentración, pues el 70 por 100 de los mismos se centra en diez países. Ahora bien, en los últimos años se ha superado el número de países en desarrollo y economías en transición (principalmente China, Federación Rusa, India, Brasil y Singapur), lo cual reduce significativamente la capacidad de impacto sobre el conjunto de países en desarrollo. Sí se tiene en cuenta que el total de entradas netas de IED a los cien países que menos reciben supone menos de un 2 por 100 del total mundial.

Los flujos netos de IED muestran una elevada volatilidad, explicada no solo por su concentración de salidas, sino por estar constituida en ocasiones hasta en un 50 por 100 por operaciones de fusiones y adquisiciones, impulsadas por la liberalización del marco regulatorio mundial de inversiones y por el proceso de internacionalización. Estas operaciones han estado empujadas principalmente por los procesos de privatización y liberalización en los países receptores y han sido llevadas a cabo a través de empresas multinacionales.

Un factor clave para explicar cómo la IED ha llegado a duplicar las tasas de crecimiento es el cambio en el esquema de financiación. Si bien inicialmente y durante los años noventa estas operaciones se realizaban fundamentalmente por intercambio de acciones, a partir de finales de esa década han pasado a realizarse a través de endeudamiento y por aplicación de los beneficios empresariales, permitiendo la entrada de importantes fondos internacionales de capital riesgo como socios capitalistas de las empresas multinacionales en sus planes de internacionalización. Este cambio ha incrementado la sensibilidad de las fusiones y adquisiciones y, por ende, de la inversión extranjera directa a los vaivenes del mercado financiero internacional, y ayuda a entender especialmente la ampliación del impacto de las fases recesivas experimentadas en los periodos de 2000-2001 y 2008-2009.

Otro factor a destacar ha sido el incremento del volumen de entradas netas de IED hacia países en desarrollo, que se ha elevado desde niveles del 1 por 100 en los años setenta a valores cercanos al 5 por 100 en los noventa y en torno al 40 por 100 a partir de 2010. Esto se explica por el incremento en la atracción de inversiones, anteriormente comentada, a países como China, India, Brasil, Federación Rusa, etcétera, en línea con la evolución del peso de los mismos dentro de la producción y comercio mundiales y por ser escenario, principalmente China o Brasil, de importantes operaciones de fusiones y adquisiciones de empresas tanto de otros países en desarrollo como de países desarrollados.

A su vez, en relación con los destinos de la IED mundial por niveles de renta, se confirma que el principal destino son los países de renta alta, si bien a lo largo de la última década se ha producido un importante incremento de los volúmenes recibidos por los países de renta media que han pasado a recibir en torno al 40 por 100 del total de la IDE mundial. Ahora bien, el porcentaje percibido por parte de las economías de renta baja continúa siendo marginal (el 2 por 100 antes indicado), mostrando la dificultad de dichos países para captar el interés inversor de las empresas multinacionales y atraer flujos de inversión privada.

\section{Metodología de estudio}

Partimos de datos recopilados de diversas fuentes, como son el Banco Mundial, el Fondo Monetario Internacional, la OCDE, la Organización Mundial del Trabajo y la $\triangleright$ 
base de datos World Development Indicators 2013 de la UNCTAD. Estos datos tienen un período de seis años, entre 2005 y 2010, y un total de 61 países que se describen en el Anexo 2.

Partiendo de las bases de datos y de la clasificación de países que se estudian, y debido a las características de las fuentes de datos empleadas en cada uno de los análisis, los datos han sido tratados como datos en panel completos y estáticos mediante modelos de regresión de componentes de error de dos factores (transversal y tiempo), incluyendo series longitudinales temporales (años) y unidades de sección cruzada o corte transversal (regiones y niveles de renta).

Los análisis se han realizado mediante modelos mixtos lineales generalizados (MMLG) de datos en panel, estimando los coeficientes del modelo mediante la técnica de mínimos cuadrados generalizados por el procedimiento de máxima verosimilitud.

La ecuación de los modelos empleados es la siguiente:

$$
Y i t=\beta O+\Sigma \beta n X i t+\mu i t
$$

Siendo:

Yit $=$ valor de la variable dependiente por unidad transversal y tiempo.

$\beta O=$ coeficiente de la intersección en el origen.

$\beta n=$ coeficiente que acompaña a cada variable independiente.

$X i t=$ valor de la variable independiente por unidad transversal y tiempo.

$\mu i t=$ término de error en el que se considera la heterogeneidad transversal no observada, el término de error clásico y los efectos temporales inobservables.
El procedimiento ha sido el estudio de la estadística descriptiva, el análisis de la distribución mediante el test de Kolmogorov Smirnoff $(p<0,01)$ con la aplicación de las necesarias transformaciones de variables en logaritmos decimales y el análisis de heterogeneidad de varianzas (heterocedasticidad) por medio de la prueba de Levene $(p<0,001)$.

Mediante el estudio de los coeficientes de correlación de Pearson se han elegido las variables independientes a incluir en el modelo, con base en la magnitud de su coeficiente de correlación $(r)$ y el nivel de significación del mismo, introduciendo dichas variables como efectos fijos y componentes principales para paliar la fuerte multicolinearidad.

Se ha realizado un primer modelo incluyendo todas las variables independientes, y a partir de ahí se han analizado los resultados obtenidos, ajustando dicho modelo y desechando diversas variables independientes debido a la persistencia de multicolinearidad y empleando como comparación del grado de ajuste de los modelos el criterio de Akaike corregido, basado en la -2log verosimilitud.

El procedimiento de introducción de datos en el modelo mixto lineal generalizado (en el sistema SPSS20) ha sido por sujeto (país), medidas repetidas (año), efectos fijos, las variables independientes como covariables y las variables nivel de renta y región como factores, todos como componentes principales para estudiar la influencia de cada uno de ellos por separado en la regresión; incluyendo la intersección en el modelo al no conocer de partida si la regresión va a pasar o no por el origen.

La hipótesis sobre si cada una de las variables independientes influyen o no sobre la variable dependiente se ha validado mediante el coeficiente $t$ para el impacto de la variable en la ecuación del modelo y mediante el nivel de $\square$ 
significación, considerado a partir de un valor $p<0,05$. Asimismo, el coeficiente que acompaña a cada una de las variables independientes de la ecuación indica la magnitud de la variación de la variable dependiente causada por cada unidad de variación de cada variable independiente, mientras el resto de variables independientes de la ecuación se mantienen constantes.

Pasamos a continuación a describir el modelo. En la construcción del modelo inicial, tal y como puede observarse en su ecuación, se han empleado 17 variables independientes seleccionadas de las cinco categorías de factores con capacidad de influencia en las entradas de inversión extranjera directa (véase Anexo 2).

Todas las variables independientes y la variable dependiente han sido transformadas en logaritmos para acercar su distribución a una normal y, además, en el caso de la variable FDL «fortaleza del sistema de derecho» de la categoría de condiciones macroeconómicas y riesgos, ésta ha sido retardada un período.

Estos índices se incluyen dentro del marco de análisis de la teoría de la localización industrial, así como parte de los factores de demanda de Porter como parte de las teorías de la organización internacional y como parte de los análisis de capacidades, tanto internacionales como españolas, entre otras, tal como lo han analizado diversos autores como Kucera (2001), Djokoto (2011) en África Subsahariana y Ranjan y Agrawal (2011) en los BRIC.

La ecuación del modelo construido es la siguiente:

$$
\begin{gathered}
\text { IED }=\beta 0+\beta 1 \text { TCOF + } \beta 2 P I B+\beta 3 I P C+ \\
+\beta 4 F D L+\beta 5 \text { TEXP + } \beta 6 \text { TIMP + } \beta 7 I M P+ \\
+\beta 8 \text { EXPOR + } \beta 9 I M P O R+\beta 10 C O M+ \\
+\beta 11 P O B+\beta 12 P I B P C+\beta 13 P A C T+ \\
+\beta 14 F B C F+\beta 15 \\
\text { RPACT + } \beta 16 C E X P+\beta 17 C I M P+\mu i t
\end{gathered}
$$

Siendo:

$\beta 0=$ coeficiente de la intersección en el origen . $\beta n(1-17)=$ coeficiente que acompaña a cada variable independiente.

$\mu i t$ = término de error en el que se considera la heterogeneidad transversal no observada, el término de error clásico y los efectos temporales inobservables.

Por otra parte, hemos construido un modelo para realizar su análisis por submuestras. Como puede observarse en su ecuación, se han empleado siete variables independientes seleccionadas de las cinco categorías de factores con capacidad de influencia en las entradas de inversión extranjera directa y cuya definición detallada puede verse en el Anexo 1.

La ecuación del modelo de submuestras construido es la siguiente:

$$
\begin{gathered}
I E D=\beta 0+\beta 1 P I B+\beta 2 I P C+\beta 3 P A C T+ \\
+\beta 4 P U R B+\beta 5 L-A G R+\beta 6 L-I M P+ \\
+\beta 7 L-C O M+\mu i t
\end{gathered}
$$

Siendo:

$\beta 0=$ coeficiente de la intersección en el origen. $\beta n(1-7)=$ coeficiente que acompaña a cada variable independiente.

$\mu i t=$ término de error en el que se considera la heterogeneidad transversal no observada, el término de error clásico y los efectos temporales inobservables.

\section{Descripción de las variables independientes relacionadas}

En el Cuadro 1 se ponen de manifiesto cuáles son las muestras de países, que se $\triangle$ 
concretan en el Anexo 1, y las variables que pasamos a comentar.

Las variables independientes seleccionadas en los modelos empleados en el contraste de la hipótesis definida están descritas en detalle en el Anexo 2. Para la elección de dichas variables independientes como factores determinantes de los flujos de entrada de inversión extranjera directa, éstas se han agrupado en cinco categorías que se definen a continuación: i) las condiciones macroeconómicas y riesgos, ii) la apertura comercial, iii) las políticas comerciales, iv) el tamaño del mercado y, finalmente, v) las capacidades, las infraestructuras y los recursos. Las especificamos a continuación:

Respecto a las condiciones macroeconómicas y riesgos a nivel general, las condiciones macroeconómicas de un país determinan el grado de incertidumbre percibido por los inversores extranjeros, de manera que un país con unas condiciones macroeconómicas estables debe recibir a priori, y considerando el carácter temporal a largo plazo de la inversión extranjera directa, un mayor volumen que un país con condiciones más volátiles.

En nuestro estudio se han elegido como índices representativos de las condiciones macroeconómicas de un país el crecimiento del PIB (porcentaje), la inflación (porcentaje), el tipo de cambio y el índice de prevalencia de derechos de propiedad, ya que han mostrado una significación importante en estudios realizados por diversos autores y en base a los cuales se espera que el crecimiento del PIB como lo estudian algunos autores como Garibaldi et al. (2002) en Europa del Este, Nonnenberg y Mendonca (2004) en los países en desarrollo, Mogrovejo (2005) en Latinoamérica, Kahai (2011) en los países en desarrollo y Ranjan y Agrawal (2011) en los BRIC (Brasil, Rusia, India y China), el tipo de cambio —variable que han utilizado otros autores como Kucera (2001) en países en desarrollo, Garibaldi et al. (2002) en Europa del Este y Kahai (2011) en los países en desarrollo- y el índice de prevalencia de derechos de propiedad -análisis realizado también por autores como Bevan and Estrin (2000) en Europa Central y del Este- hayan tenido un impacto positivo y significativo y que la inflación en cambio muestre una influencia negativa sobre las entradas de la inversión extranjera directa.

Por lo que se refiere a la segunda categoría, la apertura comercial, esta se considera como determinante de los flujos de entrada de inversión extranjera directa, ya que buena parte de la misma está orientada hacia las exportaciones y requiere en buena medida de importaciones complementarias de bienes intermedios y de capital. Dentro del conjunto de factores representativos de esta categoría, se recogen índices como el volumen de exportaciones en porcentaje sobre el PIB, el volumen de importaciones en porcentaje sobre el PIB y el volumen de comercio en porcentaje sobre el PIB, entre otros.

En el presente análisis se tendrán en cuenta los tres índices como variables independientes del modelo, ya que han mostrado una significación importante en estudios realizados por diversos autores como Holland and Pain (1998) en economías de transición, Gastanaga et al. (1998) en países en desarrollo, Nonnenberg y Mendonca (2004) en los países en desarrollo, Mogrovejo (2005) en Latinoamérica, Nunes et al. (2006) en Latinoamérica, Kahai (2011) en los países en desarrollo y Ranjan y Agrawal (2011) en los BRIC. E igualmente se espera que todos tengan una relación positiva con las entradas de IED.

La tercera de las categorías, políticas comerciales, tiene un tratamiento clásico. Los $\triangleright$ 


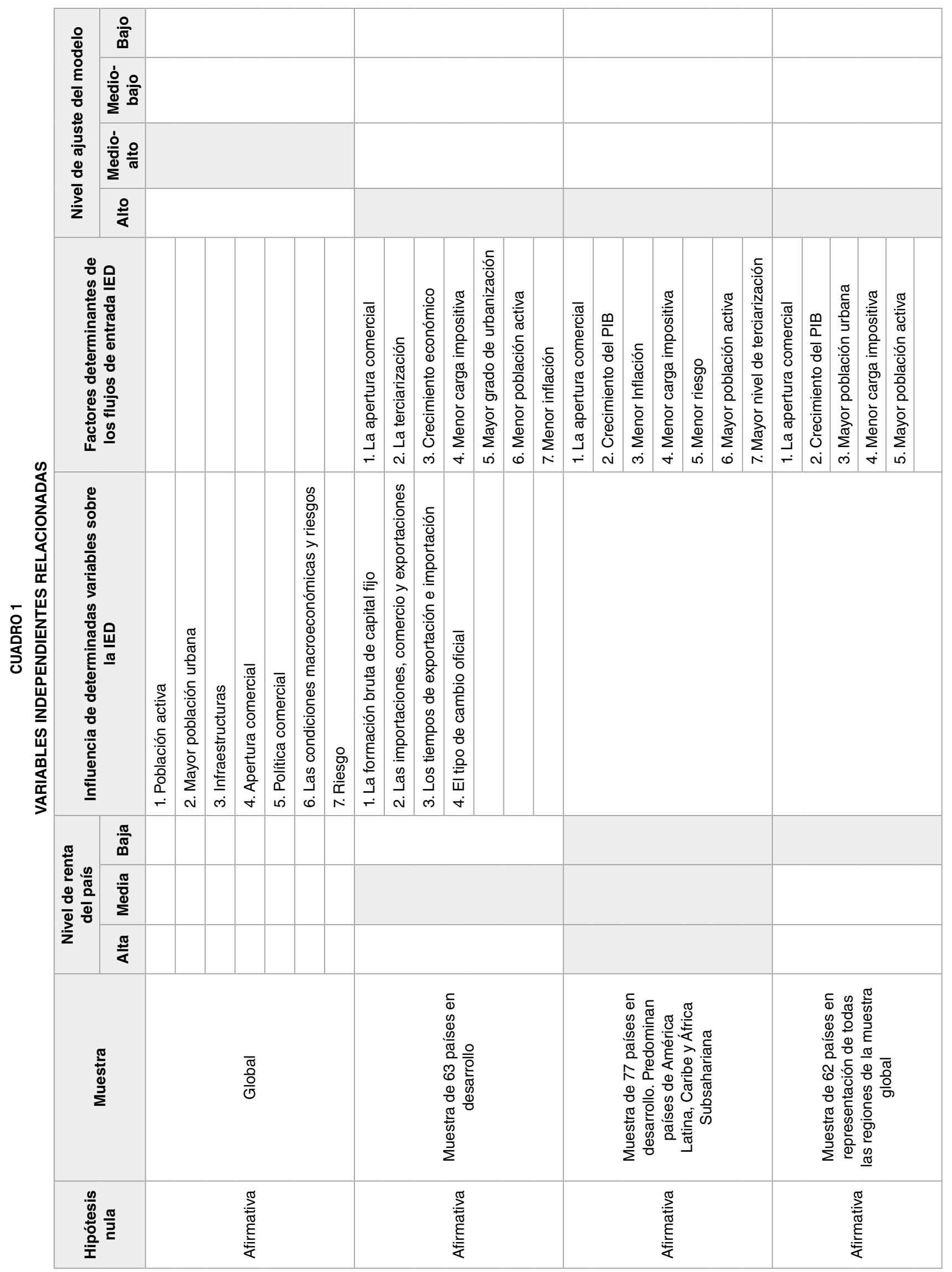




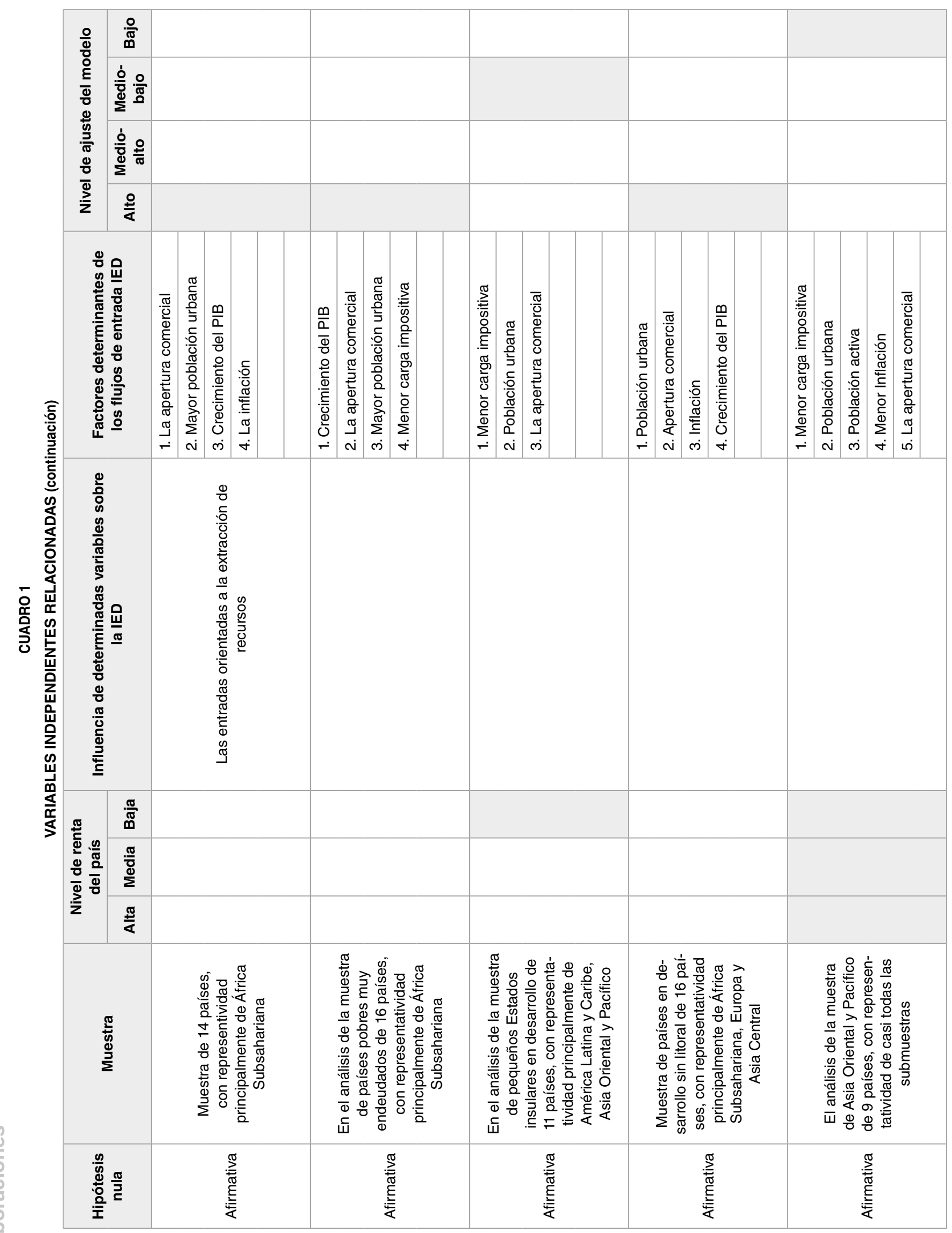




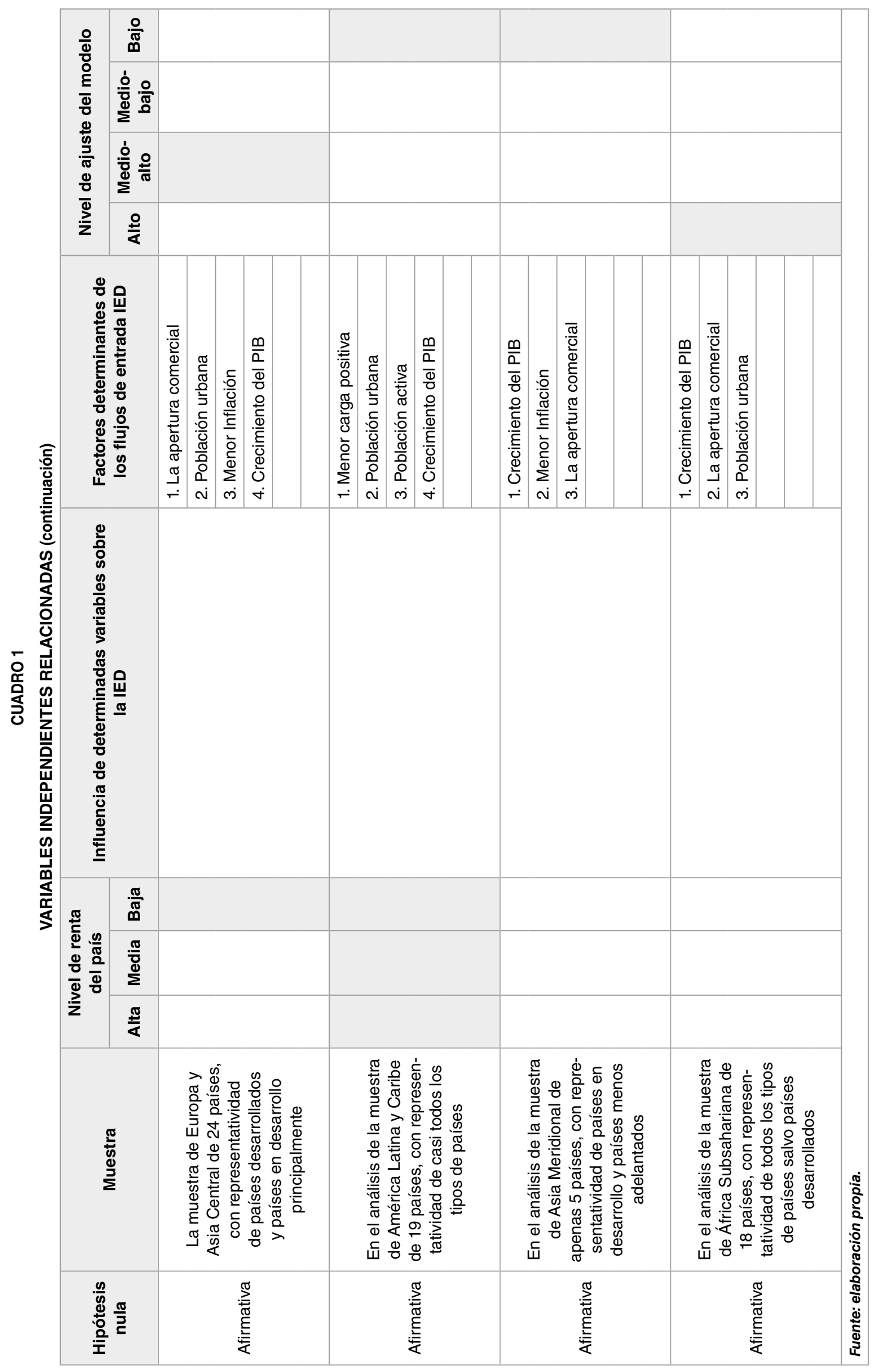


regímenes comerciales en los países receptores tienen implicaciones significativas en relación con el volumen de entradas netas de inversión extranjera directa. El grado en que los Gobiernos establecen restricciones al comercio mediante sus políticas comerciales obedece tanto a motivaciones proteccionistas de las industrias locales como a sus propios intereses electorales, si bien en última instancia dichas iniciativas restringen el comercio internacional y reducen las entradas de inversión extranjera directa, deben ser aplicadas siempre con carácter temporal y orientadas a permitir que las empresas domésticas sean competitivas internacionalmente, tal y como se establece en diversos informes internacionales, como en la Declaración de la primera Conferencia Ministerial de la OMC que se celebró en Singapur del 9 al 13 de diciembre de 1996.

Dentro del conjunto de factores representativos de esta categoría, se recogen índices como el índice de exportaciones sobre ventas, los impuestos sobre productos, el coste medio de exportación y de importación, el tiempo medio de importación y de exportación, así como diversos índices sobre políticas comerciales. Dichos índices se incluyen dentro del marco de análisis de la teoría recibida de cooperación internacional, así como parte del conjunto de variables institucionales de la escuela integradora, como también de los factores externos de política comercial de Porter como parte de las teorías de la organización internacional, entre otras.

En el presente análisis se han elegido como índices representativos de las políticas comerciales los impuestos sobre productos, el coste de importaciones, el coste de exportaciones, el tiempo de importaciones y el tiempo de exportaciones, ya que han mostrado una significación importante en estudios realizados por diversos autores como Loree and Guisinger (1995) en Estados Unidos y Gastanaga et al. (1998) en países en desarrollo.

Respecto a la cuarta de las variables, el tamaño de mercado, y la demografía a nivel general, aquel tiene una influencia significativa en la atracción de entradas de inversión extranjera directa, de acuerdo a la teoría de la localización industrial. Las empresas multinacionales tienen una motivación por localizar sus productos allí donde pueden obtener una mayor rentabilidad. Por ello, las economías con mayores mercados recibirán a priori mayores volúmenes de entrada de IED.

En el presente análisis se han elegido como índices representativos del tamaño del mercado la población total, el PIB per cápita y el porcentaje de población urbana, ya que han mostrado una significación importante en estudios realizados por diversos autores que se han citado, como Guisinger (1995) en Estados Unidos, Kucera (2001) en países en desarrollo, Garibaldi et al. (2002) en Europa del Este, Mogrovejo (2005) en Latinoamérica y Poelhekke y Van der Ploeg (2008), y en base a estos estudios se espera que todos tengan una relación positiva con las entradas de IED.

Finalmente, se han considerado como elementos de análisis las capacidades, las infraestructuras y los recursos. Con referencia a estas a nivel general y desde la perspectiva de las empresas multinacionales, origen de buena parte de las entradas de inversión extranjera directa, el acceso a recursos como la mano de obra o una infraestructura adecuada son claves tanto para el desarrollo de su actividad como para la consecución de sus objetivos en materia de productividad y eficiencia. En este sentido, un país que ofrezca unas infraestructuras de calidad y bien instaladas, así como un buen $\triangleright$ 
acceso a los recursos necesarios, obtendrá a priori un mayor volumen de entradas de IED.

Dentro del conjunto de factores representativos de esta categoría, se recogen índices como el total de fuerza de trabajo, la ratio de población activa, la red de carreteras, el uso de vehículos por habitante, el consumo de electricidad por habitante, la formación bruta de capital fijo, el porcentaje de inversión pública respecto al PIB y el peso del sector agrícola en la economía, entre otros.

En el presente análisis se han elegido como índices representativos de las capacidades las infraestructuras y los recursos, el total de fuerza de trabajo, la ratio de población activa, la formación bruta de capital fijo y el peso del sector agrícola, ya que han mostrado una significación importante en estudios realizados por diversos autores como Kucera (2001), Djokoto (2011) en Africa Subsahariana y Ranjan y Agrawal (2011) en los BRIC, y en base a los cuales se espera que ambos tengan una relación positiva con las entradas de IED.

\section{Resultados}

Según se indicó, el objetivo principal del modelo ha sido el análisis de la influencia que sobre los flujos de entradas de inversión extranjera directa en la economía han mostrado una serie de variables independientes representativas de los principales factores descriptivos de las mismas.

Para este análisis se ha establecido como hipótesis nula que la inversión extranjera directa se comporta de una manera dinámica, siendo atraída allí donde obtiene mayores ventajas y beneficios, tomando para ello una muestra global tanto a nivel regional como por tipo de países y niveles de renta.
Tanto la falta de fuentes de datos con el suficiente detalle como las limitaciones de este trabajo acotan dicho análisis de variables a nivel general, sin mostrar el detalle sectorial, por tipología de proyecto y por tipo de actividad.

Si bien entonces resulta limitada la exactitud y el detalle que se puede extraer en conclusión, tanto de las motivaciones de los flujos de entrada de inversión extranjera directa buscadas por los agentes inversores como de las estrategias de desarrollo adoptadas por los países receptores, de la misma manera dichas conclusiones resultan interesantes por servir como contraste a la hipótesis nula tanto a nivel general como por comparación entre submuestras con detalle regional y por tipo de país, lo que lo hace en sí mismo relevante.

A nivel global, el resultado obtenido tanto en el modelo inicial como en el modelo de submuestras corrobora la experiencia empírica y pone de manifiesto la existencia de una serie de factores con un nivel de influencia representativo y significativo sobre el volumen de entradas de inversión extranjera directa para el conjunto de países y durante el periodo de estudio.

El resultado obtenido del análisis del modelo inicial confirma la hipótesis nula al probar con un nivel de ajuste medio-alto la influencia de determinadas variables sobre las entradas de inversión extranjera directa, tal y como ponen de manifiesto las correlaciones observadas, especialmente en el caso de la formación bruta de capital fijo, el PIB per cápita, el volumen de población activa y el volumen de población, representativos de las capacidades, infraestructuras, recursos y del tamaño del mercado, respectivamente, y en menor medida las observadas para la apertura comercial y la política comercial, con las condiciones macroeconómicas y riesgos en último lugar. 
La muestra de 61 países expresados en el Anexo 1, principalmente países en desarrollo de renta media y de prácticamente la totalidad de regiones, así como la complejidad del modelo con 17 variables independientes, no impiden reflejar el resultado del mayor grado de influencia y significación que determinadas variables muestran dentro del modelo. De esta manera podemos inferir que las decisiones de inversión de las empresas multinacionales se ven influenciadas especialmente por: 1) la formación bruta de capital fijo, reflejo del compromiso por la creación de infraestructuras en el país de destino; 2) las importaciones, comercio y exportaciones, que facilitan tanto el abastecimiento de los insumos necesarios como la plataforma exportadora de los productos; 3 ) los tiempos de exportación e importación, que refleja el compromiso y la política de desarrollo del país receptor, así como permite mayores retornos por eficiencias y menores riesgos; $y$, finalmente, en menor medida, 4) el tipo de cambio oficial, como indicador de salud económica, estabilidad macro y menor riesgo. Esta última, en menor medida, quizás por estar circunscrita al periodo de la crisis financiera mundial.

Dentro del modelo de submuestras, en la global de 77 países, por número predominan los países en desarrollo y las regiones de América Latina, Caribe y África Subsahariana, si bien por volumen de entradas de inversión extranjera directa la mayor influencia es la de Asia Oriental y Pacífico, Europa y Asia Central, Asia Meridional y América Latina y Caribe. La existencia de países desarrollados europeos en la muestra, junto con los BRIC, tiene impacto sobre el resultado de la submuestra global, mostrando un escenario diverso, compatible con diferentes motivos, tanto de inversión extranjera directa de las empresas multinacionales origen como de estrategias de desarrollo nacionales.
Por otro lado, en el análisis de la submuestra de países en desarrollo de 62 países, con representatividad de todas las regiones de la muestra global, el nivel de ajuste del modelo es alto. El resultado confirma la hipótesis nuevamente, y en comparación con la submuestra global todas las variables independientes refuerzan su correlación con las entradas de inversión extranjera directa, a excepción del crecimiento del PIB, que se reduce ligeramente.

El grado de influencia y significación dentro del modelo muestra cómo en todos los años, y principalmente para los países de renta baja, la inversión extranjera directa se ve atraída principalmente por la apertura comercial, el crecimiento del PIB, la mayor población urbana, la menor carga impositiva y la ratio de población activa, tal como se indica en el Cuadro 1. Sin embargo, en los resultados del modelo se observa una reducción importante respecto a la submuestra global de la búsqueda del mercado interno y de eficiencias; ésta todavía predomina por el peso específico de los BRIC en la submuestra.

Al realizar el análisis de la muestra de 14 países menos adelantados, con representatividad principalmente de África Subsahariana, el resultado confirma la hipótesis nuevamente, y en comparación con la submuestra global todas las variables independientes refuerzan su correlación con las entradas de inversión extranjera directa, a excepción de la carga impositiva, que se reduce bastante.

Cabe destacar que el grado de influencia y significación dentro del modelo muestra en todos los años, aunque no en el nivel de renta, que la inversión extranjera directa se ve atraída principalmente por las variables indicadas en el Cuadro 1; refleja el crecimiento y la apertura mostrados por los países de esta región, aunque con muchas divergencias entre países. $D$ 
Las entradas de inversión extranjera directa han estado principalmente orientadas a la extracción de recursos en los países más ricos en recursos naturales, como Guinea, Mozambique, Tanzania, Sudán o Sierra Leona, y centradas principalmente en la actividad de carbón, petróleo, gas y minería, alentados por el crecimiento de la demanda global. Estos proyectos suelen ser de gran volumen y generan dependencia y riesgo de vulnerabilidad a las crisis externas.

Dentro del grupo de países como Malawi, o Uganda, aunque en menor medida, se han desarrollado inversiones en pequeños proyectos en telecomunicaciones, banca, agricultura, turismo, alimentación y bebidas y comercio. Estos proyectos, a pesar de su menor volumen, tienen más importancia en la creación de empleo (casi el 70 por 100 del total del grupo de países) y generan una mayor transferencia de habilidades. El principal desafío para el incremento de la atracción de estas entradas de inversión extranjera directa es el desarrollo de infraestructuras. Los acuerdos para establecer las concesiones son muy complejos, y no muchos países disponen de la capacidad para gestionar nuevos acuerdos en sectores altamente regulados, como son electricidad, transporte, agua, etcétera. Hasta la fecha, la mayor actividad se ha llevado a cabo en electricidad y en menor medida en transporte, en proyectos de tipo build-own-operate, buildoperate-transfer o public-private partnership.

En el análisis de la muestra de Estados pobres muy endeudados de 16 países, con representatividad principalmente de África Subsahariana, el nivel de ajuste del modelo es alto. El resultado confirma la hipótesis y es fundamentalmente similar al de la submuestra de países menos adelantados, al compartir el 68 por 100 del total de países. En comparación con la submuestra global, en este caso, todas las variables independientes refuerzan su correlación con las entradas de inversión extranjera directa, a excepción de la carga impositiva y el crecimiento del PIB.

Con la inclusión de los países de la región de América Latina y Caribe, el grado de influencia y significación dentro del modelo muestran en todos los años, aunque no en el nivel de renta, que la inversión extranjera directa se ve atraída principalmente por el crecimiento del PIB, la apertura comercial, la mayor población urbana y, en menor medida, la menor carga fiscal (Cuadro 1).

En el análisis de la muestra de pequeños Estados insulares en desarrollo de 11 países, con representatividad principalmente de América Latina y Caribe, y Asia Oriental y Pacífico, las mayores correlaciones se dan con respecto a la terciarización, la mayor población urbana y la apertura comercial principalmente.

Con la excepción principal de Singapur, el resto son economías mucho más pequeñas. La significación por años y nivel de renta es baja en el modelo.

Estos países han mostrado un crecimiento importante en las entradas de inversión extranjera directa; aunque su volumen total sea pequeño respecto al total, es alto respecto al tamaño de la economía. Por una parte, la riqueza en recursos naturales, los acuerdos preferenciales de comercio y la disponibilidad de capital humano, y en menor medida la proximidad y dependencia a la economía norteamericana de algunos de estos países, han sido los principales motivos de atracción de entradas de inversión extranjera directa a estos países, principalmente en Papúa-Nueva Guinea y Mauricio.

En el análisis de la muestra de 16 países en desarrollo sin litoral, con representatividad principalmente de África Subsahariana, Europa $\triangleright$ 
y Asia Central, el modelo muestra en todos los años que la inversión extranjera directa se ve atraída principalmente por la población urbana, y refleja la influencia de la falta de acceso al mar, la distancia a puerto y la distancia a un centro urbano. La apertura comercial impacta en la inversión extranjera directa orientada a exportaciones o intensiva en importaciones; la inflación impacta en el coste, cuya principal componente es el transporte en estos países y el crecimiento del PIB.

El tamaño de la economía y el volumen de recursos naturales juega un papel importante, como en el caso de Kazajistán por sus reservas naturales de minerales. La distancia también afecta a la inversión extranjera directa orientada a la búsqueda de eficiencias, incrementando los costes de transporte; aunque en ocasiones las políticas se orientan más a la distancia al mercado que a puerto; como en el caso de Paraguay o en Malawi, que a pesar de estar lejos de la costa cuentan con una situación privilegiada en el centro de ambos continentes.

En el análisis de la muestra de Asia Oriental y Pacífico de 9 países, con representatividad de casi todas las submuestras por tipo de país, el resultado confirma la hipótesis nuevamente, tal y como muestran las correlaciones con las entradas de inversión extranjera directa de la apertura comercial, el crecimiento del PIB y la menor inflación principalmente.

En dicha región el mayor empuje lo llevan a cabo China y Singapur, con ventajas estructurales por las políticas económicas desarrolladas en décadas pasadas y el tamaño de sus mercados internos, con un buen porcentaje de la inversión extranjera directa proveniente de la propia región. A pesar del elevado grado de intervencionismo, una buena parte de la inversión extranjera directa busca eficiencias productivas por el acceso a infraestructuras desarrolladas, personal cualificado y bajos costes laborales.

En el análisis de la muestra de Europa y Asia Central de 24 países, con representatividad de países desarrollados y países en desarrollo, principalmente, y con el nivel de ajuste del modelo medio-alto, el resultado corrobora la hipótesis nuevamente, tal y como muestran las correlaciones con las entradas de inversión extranjera directa de la apertura comercial y el crecimiento del PIB principalmente.

La principal inversión es de origen europeo y es atraída por la búsqueda de mercado interno y como plataforma para exportar, orientada principalmente a servicios.

En el análisis de la muestra de América Latina y Caribe de 19 países, con representatividad de casi todos los tipos de países, el resultado confirma la hipótesis nuevamente, tal y como muestran las correlaciones con las entradas de inversión extranjera directa de la carga impositiva, la población urbana, la apertura comercial y en menor medida el crecimiento del PIB.

La región tiene un pasado marcado por las crisis de deuda, las debilidades estructurales, la falta de infraestructura, ahorro y mercado laboral y por un aislamiento geográfico. Las principales entradas han sido en el sector servicios orientado a mercado, principalmente en Brasil, Chile y Colombia.

En el análisis de la muestra de Asia Meridional de apenas cinco países, con representatividad de países en desarrollo y otros menos adelantados, principalmente, el modelo arroja que la inversión extranjera directa se ve atraída por una menor inflación, menor carga impositiva y un mayor crecimiento del PIB.

Cabe destacar que la región ha sobrellevado un proceso de reducción de aranceles y de eliminación de barreras al comercio $D$ 
muy importante, principalmente en el sector de servicios tecnológicos, como servicios de tecnologías de la información y servicios empresariales en India, además de otros como biotecnología, química y farmacia. Actualmente, India vive una tendencia de progresiva apertura a la inversión extranjera directa.

Por último, en el análisis de la muestra de África Subsahariana de 18 países, con representatividad de todos los tipos de países salvo países desarrollados, el resultado confirma la hipótesis nuevamente, tal y como muestran las correlaciones con las entradas de inversión extranjera directa de la población urbana, la apertura comercial, la terciarización de la economía y el crecimiento del PIB.

El crecimiento de las entradas de inversión extranjera directa a la región se ha producido por la mejora de las condiciones económicas y la mayor apertura al exterior, con numerosos acuerdos internacionales de inversión y doble imposición. El origen principal de la inversión ha sido EEUU y Europa, orientada principalmente a la búsqueda de recursos naturales.

\section{Bibliografía}

[1] BEVAN, A. A. y ESTRIN, S. (2000). Patterns of foreign direct investment and trade in central and eastern europe, mimeo.

[2] DJOKOTO, J. G. (2011). «Inward Foreign Direct Investment Flows, Growth, and Agriculture in Ghana: A Granger Causal Analysis». International Journal of Economics and Finance, vol. 3, n.ำ 6 .

[3] FONDO MONETARIO INTERNACIONAL (2009). Balance of Payments and International Investment Position Manual, Sixth Edition (BPM6), New York.

[4] GARIBALDI, P.; MORA. N.; SAHAY. R. y ZETTELMEYER, J. (2002). «What moves capital to transition economies?». IMF working paper WP/02/64.
[5] GASTANAGA, V. M.; NUGENT, J. B. y PASHAMOVA, B. (1998). «Host Country Reforms and FDI Inflows: How Much Difference Do They Make? », World Development, vol. 26, n. -7 , pp. 1299-1314.

[6] HOLLAND, D. y PAIN, N. (1998). «The determinants and impact of foreign direct investment in the transition economies: a panel data analysis». National Institute of Economic and Social Research.

[7] KAHAI, S. K. (2011). «Traditional And NonTraditional Determinants Of Foreign Direct Investment In Developing Countries». Journal Of Applied Business Research, vol. 20, n. 1 .

[8] KUCERA, D. (2001). «The effects of core workers rights on labour costs and foreign direct investment: Evaluating the conventional wisdom». IILS. Discussion paper, DP.130. 2001.

[9] LOREE, D. W. y GUISINGER, S. E. (1995). «Policy and non policy determinants of U.S equity foreign direct investment». Journal of International Business Studies, Second Quarter, pp. 281- 299.

[10] MOGROVEJO, J. A. (2005). «Factores determinantes de la inversión extranjera directa en algunos países de Latinoamérica». Estudios Económicos de Desarrollo Internacional. AEEADE, vol. 5-2.

[11] NONNENBERG, M. y MENDONCA, M. (2004). «The determinants of direct foreign investment in developing countries». January. Working Paper, Institute of Applied Economic Research.

[12] NUNES, C. L.; OSCATEGUI, J. y PESCHIERA, J. (2006). «Determinants of FDI in Latin America». Documento de trabajo, n. 252.

[13] ORGANIZACIÓN MUNDIAL DEL COMERCIO (1996). Declaración de la primera Conferencia Ministerial. Singapur.

[14] POELHEKKE, S. y VAN DER PLOEG, F. (2008). «Growth, Foreign Direct Investment and Urban Concentrations: Unbundling Spatial Lags». De Nederlandsche Bank NV Working Paper, n.ำ 195.

[15] RANJAN, V. y AGRAWAL, G. (2011). «FDI Inflow Determinants in BRIC countries: A Panel Data Analysis». International Business Research, vol. 4, n. 4 . 


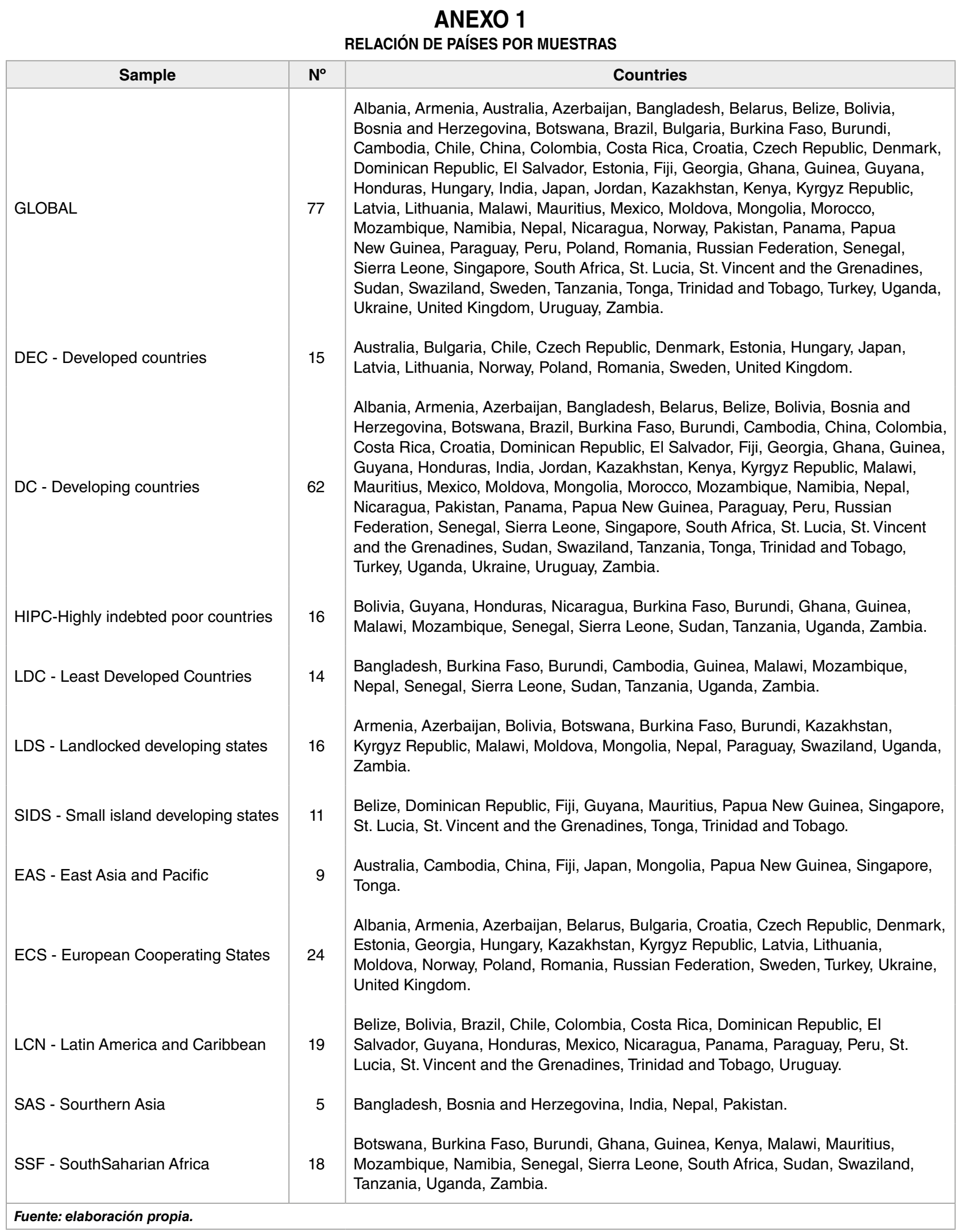


ANEXO 2

DESCRIPCIÓN DE LAS VARIABLES INCLUIDAS EN EL MODELO

\begin{tabular}{|c|c|c|}
\hline Variable & Descripción & Fuente \\
\hline IED & $\begin{array}{l}\text { Foreign direct investment, net } \\
\text { inflows (\% of GDP) }\end{array}$ & $\begin{array}{l}\text { WDI } 2013 \text { (BX.KLT.DINV.WD.GD.ZS). Source: International Monetary Fund, } \\
\text { International Financial Statistics and Balance of Payments databases, World Bank, } \\
\text { Global Development Finance, and World Bank and OECD GDP estimates. }\end{array}$ \\
\hline Crec PIB & GDP growth (annual \%) & $\begin{array}{l}\text { WDI } 2013 \text { (NY.GDP.MKTP.KD.ZG). Source: World Bank national accounts data, and } \\
\text { OECD National Accounts data files. }\end{array}$ \\
\hline IPC & $\begin{array}{l}\text { Inflation, consumer prices } \\
\text { (annual \%) }\end{array}$ & $\begin{array}{l}\text { WDI } 2013 \text { (FP.CPI.TOTL.ZG). Source: International Monetary Fund, International } \\
\text { Financial Statistics and data files. }\end{array}$ \\
\hline RPACT & $\begin{array}{l}\text { Labor participation rate, total } \\
(\% \text { of total population ages } 15+)\end{array}$ & $\begin{array}{l}\text { WDI } 2013 \text { (SL.TLF.CACT.ZS). Source: International Labour Organization, Key } \\
\text { Indicators of the Labour Market database. }\end{array}$ \\
\hline PURB & Urban population ( $\%$ of total) & $\begin{array}{l}\text { WDI } 2013 \text { (SP.URB.TOTL.IN.ZS). Source: United Nations, World Urbanization } \\
\text { Prospects. }\end{array}$ \\
\hline L-AGR & $\begin{array}{l}\text { Agriculture, value added } \\
(\% \text { of GDP) }\end{array}$ & $\begin{array}{l}\text { WDI } 2013 \text { (NV.AGR.TOTL.ZS). Source: World Bank national accounts data, and } \\
\text { OECD National Accounts data files. }\end{array}$ \\
\hline L-IMP & $\begin{array}{l}\text { Net taxes on products (current } \\
\text { LCU) }\end{array}$ & $\begin{array}{l}\text { WDI } 2013 \text { (NY.TAX.NIND.CN). Source: World Bank national accounts data, and OECD } \\
\text { National Accounts data files. }\end{array}$ \\
\hline L-COM & Trade ( $\%$ of GDP) & $\begin{array}{l}\text { WDI } 2013 \text { (NE.TRD.GNFS.ZS). Source: World Bank national accounts data, and } \\
\text { OECD National Accounts data files. }\end{array}$ \\
\hline
\end{tabular}

ANEXO 3

APLICACIÓN ESTADISTICA

\begin{tabular}{|c|c|c|c|c|c|c|c|c|}
\hline Valores & Intersección & CREC PIB & IPC & RPACT & PURB & L-AGR & L-IMP & L-COM \\
\hline \multicolumn{9}{|c|}{ GLOBAL - - Akaike: 4933.53} \\
\hline Correlation - C & & ,204** & $-0,049$ &,$- 129^{\star *}$ & $156^{\star *}$ &,$- 237^{\star *}$ &,$- 196^{\star *}$ & $399^{\star *}$ \\
\hline Coefficient - c & $-8,178$ & 0,228 & 0 & 0,045 & 0,015 & $-0,757$ & $-0,31$ & 6,79 \\
\hline T-student - t-s & $-3,037$ & 6,905 & $-2,449$ & 2,42 & 1,509 & $-1,278$ & $-2,598$ & 9,11 \\
\hline Significance - S & 0,002 & 0 & 0,015 & 0,016 & 0,13 & 0,202 & 0,01 & 0 \\
\hline \multicolumn{9}{|c|}{ DEC - Developed countries - Akaike: 215.59} \\
\hline Correlation - C & &, $316^{\star \star}$ &, $232^{\star *}$ &,$- 320^{\star *}$ &,$- 257^{* \star}$ &, $398^{\star \star}$ &,$- 230^{\star *}$ & $608^{\star *}$ \\
\hline Coefficient - c & $-2,84$ & 0,002 & 0 & $-0,012$ & 0,01 & 0,34 & $-0,007$ & 1,42 \\
\hline T-student - t-s & $-3,057$ & 2,12 & 1,65 & $-1,22$ & 3,29 & 1,95 & $-0,21$ & 8,497 \\
\hline Significance - S & 0,003 & 0,03 & 0,1 & 0,22 & 0,001 & 0,05 & 0,83 & 0 \\
\hline \multicolumn{9}{|c|}{ DC - Developing countries - Akaike: 1041.481} \\
\hline Correlation - C & &, $154^{\star \star}$ & $-0,049$ &,$- 208^{\star *}$ &, $302^{\star \star}$ &,$- 322^{\star \star}$ &,$- 209^{\star \star}$ &, $412^{\star \star}$ \\
\hline Coefficient - c & $-1,188$ & 0,02 & 0 & 0,004 & 0,005 & 0,044 & $-0,038$ & 0,828 \\
\hline T-student - t-s & $-3,35$ & 4,24 & $-1,517$ & 1,73 & 3,586 & 0,525 & $-2,33$ & 7,65 \\
\hline Significance - S & 0,001 & 0 & 0,13 & 0,08 & 0 & 0,6 & 0,02 & 0 \\
\hline \multicolumn{9}{|c|}{ LDC - Least Developed Countries - Akaike: 359.627} \\
\hline Correlation - C & &, $264^{\star \star}$ &, $167^{*}$ &,$- 174^{\star}$ &, $441^{* \star}$ &,$- 222^{\star \star}$ & 0,011 & $447^{\star \star}$ \\
\hline Coefficient - c & $-3,945$ & 0,071 & 0 & $-0,017$ & 0,032 & $-0,148$ & 0,059 & 1,996 \\
\hline T-student - t-s & $-3,63$ & 3,758 & 2,486 & $-2,31$ & 4,683 & $-0,338$ & 0,9 & 5,356 \\
\hline Significance - S & 0 & 0 & 0,014 & 0,02 & 0 & 0,736 & 0,37 & 0 \\
\hline \multicolumn{9}{|c|}{ HIPC-Highly indebted poor countries - Akaike: 382.032} \\
\hline Correlation - C & & 0,129 & 0,112 &,$- 316^{\star *}$ &, $403^{\star \star}$ &,$- 313^{\star *}$ & $-0,121$ & $419^{* *}$ \\
\hline Coefficient - c & $-3,49$ & 0,077 & 0 & $-0,014$ & 0,016 & 0,174 & 0,109 & 1,356 \\
\hline T-student - t-s & $-2,79$ & 4,79 & 2,287 & $-2,326$ & 3,312 & 0,423 & 2,508 & 4,493 \\
\hline Significance - S & 0,006 & 0 & 0,024 & 0,021 & 0,001 & 0,673 & 0,013 & 0 \\
\hline
\end{tabular}


ANEXO 3

APLICACIÓN ESTADÍSTICA (continuación)

\begin{tabular}{|c|c|c|c|c|c|c|c|c|}
\hline Valores & Intersección & CREC PIB & IPC & RPACT & PURB & L-AGR & L-IMP & L-COM \\
\hline \multicolumn{9}{|c|}{ SIDS - Small island developing states - Akaike: 188.537} \\
\hline Correlation - C & & 0,129 & $-0,146$ & 0,047 &, $308^{* *}$ &,$- 419^{\star \star}$ & 0,031 & ,238* \\
\hline Coefficient - c & 1,765 & 0 & 0 & $-0,002$ & 0,005 & 0,025 & $-0,226$ & 0,553 \\
\hline T-student - t-s & 1,788 & 0,018 & $-0,682$ & $-0,212$ & 1,869 & 0,102 & $-3,599$ & 1,75 \\
\hline Significance - S & 0,077 & 0,985 & 0,497 & 0,833 & 0,065 & 0,919 & 0,001 & 0,083 \\
\hline \multicolumn{9}{|c|}{ LDS - Landlocked developing states - Akaike: 400.414} \\
\hline Correlation - C & &, $263^{\star *}$ & 0,128 &,$- 466^{\star \star}$ &, $514^{\star \star}$ &,$- 438^{\star \star}$ &,$- 176^{\star}$ &, $570^{\star *}$ \\
\hline Coefficient - c & $-1,908$ & 0,021 & 0 & $-0,014$ & 0,013 & $-0,686$ & 0,103 & 1,12 \\
\hline T-student - t-s & $-1,53$ & 2,166 & 2,515 & $-1,88$ & 3,05 & $-2,12$ & 2,017 & 2,75 \\
\hline Significance - S & 0,128 & 0,032 & 0,013 & 0,062 & 0,003 & 0,035 & 0,045 & 0,007 \\
\hline \multicolumn{9}{|c|}{ EAS - East Asia and Pacific - Akaike: 116.282} \\
\hline Correlation - C & &, $337^{\star \star}$ & ,256* & 0,108 & 0,104 & $-0,147$ & $-0,051$ & $605^{\star \star}$ \\
\hline Coefficient - c & $-7,89$ & 0,02 & 0 & 0,179 & 0,084 & $-0,196$ & $-0,495$ & $-1,456$ \\
\hline T-student - t-s & $-5,808$ & 2,077 & 3,479 & 6,166 & 9,408 & $-1,095$ & $-9,794$ & $-3,157$ \\
\hline Significance - S & 0 & 0,041 & 0,001 & 0 & 0 & 0,277 & 0 & 0,002 \\
\hline \multicolumn{9}{|c|}{ ECS - European Cooperating States - Akaike: 278.511} \\
\hline Correlation - C & &, $247^{\star \star}$ &,$- 193^{\star \star}$ & 0,047 &,$- 194^{\star \star}$ & 0,077 &,$- 195^{\star \star}$ &, $321^{\star \star}$ \\
\hline Coefficient - c & $-0,484$ & 0,014 & 0 & 0,007 & $-0,011$ & $-0,174$ & 0,028 & 0,948 \\
\hline T-student - t-s & $-0,892$ & 2,93 & $-3,179$ & 1,56 & $-3,557$ & $-1,29$ & $-1,23$ & 5,807 \\
\hline Significance - S & 0,373 & 0,004 & 0,002 & 0,12 & 0 & 0,198 & 0,22 & 0 \\
\hline \multicolumn{9}{|c|}{ SAS - Sourthern Asia - Akaike: 79.498} \\
\hline Correlation - C & & 0,168 & 0,019 &,$- 760^{\star *}$ &, $768^{\star \star}$ &,$- 711^{\star *}$ & $-0,173$ & $462^{\star \star}$ \\
\hline Coefficient - c & $-2,953$ & 0,083 & 0 & $-0,02$ & 0,029 & 0,248 & 0,056 & 0,98 \\
\hline T-student - t-s & $-0,885$ & 5,197 & 3,7 & $-1,139$ & 1,315 & 0,331 & 0,6 & 1,77 \\
\hline Significance - S & 0,38 & 0 & 0,001 & 0,26 & 0,196 & 0,742 & 0,549 & 0,083 \\
\hline \multicolumn{9}{|c|}{ SSF - SouthSaharian Africa - Akaike: 439.804} \\
\hline Correlation - C & &, $192^{\star \star}$ &, $163^{\star}$ & $-0,133$ &, $361^{\star *}$ &,$- 199^{\star *}$ &,$- 158^{\star}$ &, $353^{\star \star}$ \\
\hline Coefficient - c & $-3,874$ & 0,065 & 0 & 0,003 & 0,018 & 0,211 & 0,035 & 1,27 \\
\hline T-student - t-s & $-3,885$ & 4,015 & 1,419 & 0,54 & 3,76 & 0,787 & 0,86 & 3,875 \\
\hline Significance - S & 0 & 0 & 0,158 & 0,59 & 0 & 0,432 & 0,389 & 0 \\
\hline \multicolumn{9}{|c|}{ LCN - Latin America and Caribbean - Akaike: 100.905} \\
\hline Correlation - C & &, $191^{\star \star}$ & $-0,126$ &,$- 156^{\star}$ &,$- 369^{\star *}$ &,$- 186^{\star *}$ &,$- 383^{\star *}$ &, $343^{* *}$ \\
\hline Coefficient - c & 2,46 & 0,016 & 0 & $-0,017$ & $-0,006$ & 0,02 & $-0,066$ & 0,215 \\
\hline T-student - t-s & 4,479 & 3,28 & $-0,855$ & $-3,7$ & $-3,88$ & 0,148 & $-4,28$ & 1,67 \\
\hline Significance - S & 0 & 0,001 & 0,394 & 0 & 0 & 0,883 & 0 & 0,097 \\
\hline \multicolumn{9}{|c|}{ Fuente: elaboración propia. } \\
\hline
\end{tabular}

